\title{
CONCENTRACIONES Y COMPORTAMIENTO DE METALES PESADOS EN UNA ZONA ESTUARINA DE VENEZUELA
}

\author{
ARISTIDE MÁRQUEZ, WILLIAM SENIOR \\ y GREGORIO MARTÍNEZ
}

xiste una amplia gama
de productos derivados nas que, una vez vertidos a los ríos, costas y estuarios, causan alteraciones ecológicas especificas. Tal es el caso de los metales pesados. El Grupo de Expertos Sobre Aspectos Científicos de Contaminación Marina (GESAM) advierte sobre la imperativa y urgente necesidad de crear programas de seguimiento continuo en los ríos, estuarios y zonas costeras, para obtener información que permita diseñar planes de control, ordenación, manejo y uso de esos cuerpos de aguas (David y Phillips, 1995; Salinas et al.,1996). La presencia de elementos metálicos en sistemas acuáticos fluviales y costeros se origina por las interacciones del agua con los sedimentos y la atmósfera con la que está en contacto, produciéndose fluctuaciones en las concentraciones en el agua, como resultado de las fuerzas hidrodinámica naturales, biológicas y químicas (Rainbow, 1995).

El río Manzanares constituye una cuenca aislada perteneciente a

Nota. Este trabajo forma parte de la Tesis de Lic. en Química realizada en el Departamento de Química de la UDO. Sucre, por el Lic. Aristide Márquez en el año 1997. la gran cuenca del Mar Caribe, caracterizada por la existencia de un período de sequía, de diciembre a junio, y uno de lluvia de julio a noviembre. El río nace en el cerro Turimiquire, a una altura superior a $2.000 \mathrm{~m}$ sobre el nivel del mar, desemboca en la entrada del Golfo de Cariaco (Figura 1), y ejerce gran influencia hacia el lado oeste de la costa de Cumaná, ubicada entre $10^{\circ}$ y $10^{\circ} 30^{\prime} \mathrm{de}$ latitud Norte, y 64' $10^{\prime}$ y $64^{\circ} 20^{\prime}$ de longitud Oeste. Su hoya hidrográfica cubre una extensión aproximada de 165.210 hectáreas y el aporte anual de su escorrentía se estima en 600 millones de $\mathrm{m}^{3}$, que se reparte entre la zona del aliviadero, sector donde se produce una gran sedimentación, y su desembocadura original. En los últimos años (1980-1991) el aporte se ha incrementado en más de un $20 \%$, a $770.689 \times 10^{6} \mathrm{~m}^{3}$ (Aguilera y Rojas,1976; Alvarado, 1976; Senior, 1994; Márquez, 1997).

El río funciona como una especie de laguna de oxidación natural, debido al alto grado de contaminación que presentan sus aguas (Fernández, 1984). Desde su cuenca alta recibe las descargas de desechos químicos de la central azucarera de Cumanacoa, Distrito Montes; de las aguas domésticas de la región, que son vertidas al río Guasdua, afluente del Manzanares; y de los com- puestos químicos utilizados en las prácticas agrícolas (Senior, 1994; León, 1995). Al mismo tiempo, cerca de su desembocadura recibe las descargas industriales de una procesadora de pescado, y los desechos sólidos y líquidos de un astillero naval y del Mercado Municipal de la ciudad de Cumaná. Estudios efectuados en la década de los noventa evidencian la alteración de la calidad de las aguas de este río (Senior y Godoy, 1991; Iabichella, 1993; León, 1995), hecho que pone de manifiesto la necesidad de un seguimiento continuo de sus aguas.

En vista del interés reinante por la contaminación de metales pesados en los sistemas estuarinos y zonas litorales, se presentan los resultados obtenidos en las concentraciones y en el comportamiento de los metales $\mathrm{Fe}, \mathrm{Mn}$, $\mathrm{Cd}, \mathrm{Zn}, \mathrm{Pb}, \mathrm{Cu}$, Ni y $\mathrm{Cr}$ en los sólidos suspendidos de las aguas superficiales del río Manzanares, Estado Sucre, Venezuela durante el año 1997.

\section{Materiales y Métodos}

Un total de 22 muestras de agua superficial fueron recolectadas mensualmente en el río, durante el año 1997. En este trabajo se presentan los diagramas de mezcla metal - salinidad para los meses de marzo (aguas bajas) y

PALABRAS CLAVES / Metales Pesados / Comportamiento / Contaminación de Aguas /

Aristide Márquez. Licenciado en Química. Profesor-Investigador, Coordinador del Laboratorio de Metales Pesados, División de Recursos Acuáticos. Dirección: Instituto Limnológico, Universidad de Oriente (UDO), Caicara del Orinoco, Estado Bolívar, Venezuela. e-mail: iludo@cantv.net

William Senior. Doctor en Química. Profesor-Investigador, Jefe del Departamento de Oceanografía, Instituto Oceanográfico de Venezuela, UDO. Dirección: Departamento de Oceanografía, I.O.V, Universidad de Oriente. Av. Universidad, Cerro Colorado, A.P.245,6001, Cumaná, Venezuela. E-mail: Wsenior@sucre.udo.edu.ve

Gregorio Martínez. MsC. en Ciencias Marinas. Profesor-Investigador, Coordinador del Laboratorio de Metales Pesados, Instituto Oceanográfico de Venezuela (UDO). 
agosto (aguas altas) por ser meses en los cuales todos los metales evaluados estuvieron presentes y fueron evaluados a diferentes salinidades. La toma de muestras se realizó siguiendo el gradiente salino hasta alcanzar salinidades superiores a 36 psu (unidades practicas de salinidad), en dirección a la Península de Araya (Figura 1). La salinidad fue medida in situ en un salinómetro portátil YSI modelo 33 con precisión de $\pm 0,1 \mathrm{psu}$, y confirmada en uno de inducción Kahlsico modelo 118 WC 200, con precisión $\pm 0,001$ psu. Todo el material de vidrio y plástico destinado para la toma de muestras fue previamente lavado con ácido nítrico al $10 \%$ v/v. Un litro de cada muestra fue recolectado en envases de poliestireno y se filtraron inmediatamente en un equipo Millipore, utilizando membranas filtrantes de celulosa tipo HA con poros de $0,45 \mu \mathrm{m}$ de diámetro, las cuales eran previamente lavadas con una solución de ácido clorhídrico diluido y agua desionizada. El material retenido en el filtro fue tratado con una mezcla de ácidos nítrico y clorhídrico concentrados para obtener los metales presentes en fase acuosa (Greenber et al., 1992). El extracto fue analizado por Espectrofotometría de Absorción Atómica con llama de aire - acetileno, en un equipo Perkin - Elmer modelo 3110 con corrector de fondo de deuterio. Los blancos recibieron el mismo tratamiento. En la Tabla I se presentan la precisión y reproducibilidad para cada uno de los metales evaluados, obtenida mediante la determinación de cinco replicas de una muestra. El material en suspensión fue retenido a través de filtros de vidrio tipo A/E $47 \mathrm{~mm}$. La precisión de este método es de $\pm 0,15 \mathrm{mg} / \mathrm{l}$ y proporciona un limite de detección de 0,3 mg depositados en el filtro (Senior, 1995). Las concentraciones de metales permitidas por la legislación venezolana que se citan en el texto son tomadas de la Gaceta Oficial No 34.829 del 29/01/1992.

Para el análisis de difracción de rayos $\mathrm{X}$, el material en suspensión retenido en el filtro de $0,45 \mu \mathrm{m}$ fue secado en estufa a $80^{\circ} \mathrm{C}$, macerado y homogenizado. Los análisis fueron realizados en el Departamento de Química de la Universidad de Oriente, Núcleo de Sucre, mediante un difractómetro Siemens modelo D5000.

\section{Resultados y Discusión}

El comportamiento de los metales se interpretó de acuerdo al índice de conservatividad propuesto por Liss (1976), mediante diagramas que indican la relación de mezcla entre las concentraciones de los metales y la salinidad.

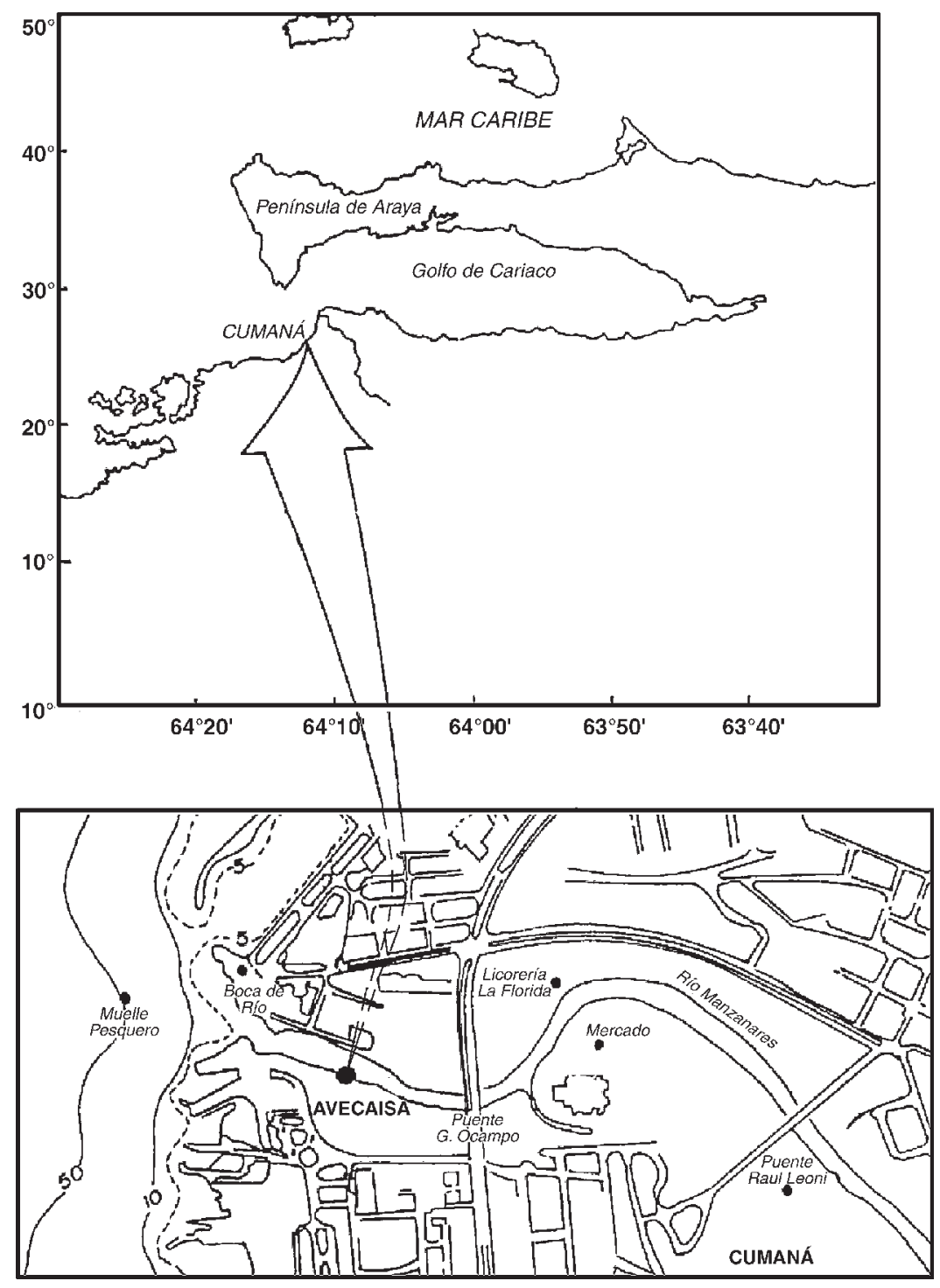

Figura 1. Área de estudio.

Las concentraciones máximas de los ocho metales estudiados y las remociones encontradas en el período de aguas bajas (febrero - junio) y altas (agosto - noviembre), se reportan en la Tabla II. El comportamiento presentado por los mismos es representado en las Figuras $2 \mathrm{a}-\mathrm{h}$ para los meses de marzo (aguas bajas) y agosto (aguas altas). Todos los elementos presentaron un comportamiento no conservativo con remoción a bajas salinidades ( $<10$ psu). Los valores para el material en suspensión se incluyen en la Tabla II y el comportamiento de éste se presenta en la Figura 4.

Las remociones fueron calculadas a partir de los diagramas de mezcla metal-salinidad de cada metal durante cada mes de muestreo, restando el valor de la concentración mayor $(\mathrm{C} 1)$ en el inicio del mezclado del agua fluvial con la marina con el valor en donde se observa el decaimiento de la misma (C2) en el diagrama de mezcla, dividiendo luego esta sustracción entre el valor mayor (C1) y multiplicándolo por 100 (Figura 3).

Las concentraciones de hierro encontradas oscilaron entre 123 y $405 \mu \mathrm{mol} / \mathrm{l}$, con remociones entre 12 y $53 \%$ y 15 a $67 \%$ para los períodos de aguas bajas y altas respectivamente, obteniéndose las máximas concentraciones en el mes de agosto. En la Figura 2a se observa que los aportes de Fe incrementan en el período de mayor gasto del río, determinándose una relación positiva altamente significativa $(r>0,90)$ con el material en suspensión. A pesar de que el Fe presentó un comportamiento no conservativo durante todo el estudio, en agosto se comporto cuasi-conservativo $(\mathrm{r}=0,9637)$, posiblemente por la mezcla 
TABLA I

REPRODUCIBILIDAD Y PRECISIÓN DE LAS DETERMINACIONES DE METALES REALIZADAS EN LA FRACCIÓN SUSPENDIDA EN EL PRESENTE ESTUDIO.

\begin{tabular}{lrccccccc}
\hline & $\mathrm{Fe}$ & $\mathrm{Mn}$ & $\mathrm{Zn}$ & $\mathrm{Cr}$ & $\mathrm{Cd}$ & $\mathrm{Pb}$ & $\mathrm{Cu}$ & $\mathrm{Ni}$ \\
\hline Promedio $(\mu \mathrm{mol} / \mathrm{l})$ & 72,663 & 0,923 & 0,193 & 0,043 & 0,025 & 0,055 & 0,052 & 0,093 \\
Desviación St. & 0,019 & 0,003 & 0,001 & 0,001 & 0,001 & 0,0002 & 0,001 & 0,002 \\
Precisión & 0,018 & 0,019 & 0,238 & 1,212 & 1,099 & 0,005 & 0,775 & 0,537 \\
\hline
\end{tabular}

casi total de las aguas del río con las del mar. La legislación venezolana establece concentraciones limites de $179 \mu \mathrm{mol} / \mathrm{l}$ para las aguas de descargas, y 5,36 $\mu \mathrm{mol} / \mathrm{l}$ para las aguas destinadas al consumo humano.

Los altos valores encontrados y la alta correlación con el material en suspensión sugieren que este elemento no esta siendo removido de los sólidos suspendidos durante el proceso de mezcla, y por ende el comportamiento y la remoción presentada son exclusivos de los procesos de sedimentación de los sólidos suspendidos en forma de oxihidróxidos coloidales y precipitación debido al cambio iónico en los primeros inicios de la mezcla del agua fluvial con el agua de mar. Sin embargo los efectos de cambios en las cantidades del material suspendido por volumen de agua no se descartan. Wells y Meyer (1991) señalan que en los medios bajo la influencia de los ríos el hierro está asociado preferencialmente al material suspendido. Martin et al. (1993) y Zhang (1995) de igual manera indican que la remoción del Fe a bajas salinidades ocurre por la floculación y precipitación debidos a los cambios en el pH y la fuerza iónica
Los aportes de manganeso al río variaron entre $1,80 \mu \mathrm{mol} / \mathrm{l}$ en la época de sequía y $5,55 \mu \mathrm{mol} / 1$ en el período de lluvia. Los valores mayores se encontraron en el período de mayor caudal del río, coincidiendo con las concentraciones máximas de $\mathrm{Fe}$, debido a que en este mes el río arrastró una gran carga sedimentaria $(880 \mathrm{mg} / \mathrm{l})$. Los diagramas de mezcla del Mn en función de la salinidad (Figura 2b) para agosto reflejan un máximo en las concentraciones a salinidad entre 0 y 2 psu. El Mn presentó remociones entre 16 - $67 \%$ en la época de sequía y 26 - 55\% en la de lluvia. El nivel de Mn permitido por la legislación venezolana para aguas destinadas al consumo es $0,91 \mu \mathrm{mol} / 1$.

La alta correlación presentada entre este metal y el $\mathrm{Fe}$ $(\mathrm{r}=0,9064)$, así como con el material en suspensión ( $\mathrm{r}=0,8950)$, en el mes de agosto, indica que puede estar adsorbido a las partículas de $\mathrm{Fe}$ y/o coprecipitado, o que puede tener la misma fuente de origen que éste. En ambientes acuáticos el $\mathrm{Mn}$ disuelto es altamente reactivo y presenta una alta capacidad de adsorción al $\mathrm{Fe}$ y al material suspendido, en forma de óxidos coloidales, lo cual contribuye al mismo tiempo en el comportamiento de otros elementos dentro de los estuarios (Duinker y Nolting, 1978; Morris and Bale, 1979). Por estar asociado el $\mathrm{Mn}$ al $\mathrm{Fe}$, su comportamiento depende igualmente de los procesos que rigen el comportamiento de éste. Este comportamiento y observaciones similares han sido reportados en la bahía de Chesapeake (Carpenter et al., 1975) y en el río Amazonas (Gibbs, 1977).

Las concentraciones de cobre en aguas naturales están por debajo de 0,39 $\mu \mathrm{mol} / 1$ (Landing et al., 1992). En el presente estudio, las mismas variaron de 0,09 $\mu \mathrm{mol} / 1$ en el período de aguas bajas hasta valores de $0,71 \mu \mathrm{mol} / 1$ en los meses de mayor caudal, alcanzándose el máximo en agosto, como ocurre con el $\mathrm{Fe}$ y el $\mathrm{Mn}$. Los valores de $\mathrm{Cu}$ admitidos por la legislación venezolana tienen limites de $1,6 \times 10^{-3} \mu \mathrm{mol} / 1$ para las aguas descargadas al medio marino - costero y valores similares para las aguas de consumo humano (Senior,1994). Se observó poca variabilidad temporal de las concentraciones de $\mathrm{Cu}$ durante los meses intermedios a los meses extremos de períodos de aguas bajas y altas, exceptuando agosto, cuando se alcanzaron los máximos ni-

TABLA II

CONCENTRACIONES MÁXIMAS ( $\mu$ mol/1) Y \% DE REMOCIÓN EN LA FRACCIÓN SUSPENDIDA

DE LAS AGUAS ESTUARINAS DEL RÍO MANZANARES, VENEZUELA, EN LOS PERÍODOS DE AGUAS BAJAS (FEBRERO - JUNIO) Y ALTAS (AGOSTO - NOVIEMBRE).

\begin{tabular}{|c|c|c|c|c|c|c|c|c|c|}
\hline & $\mathrm{Fe}$ & $\mathrm{Mn}$ & $\mathrm{Zn}$ & $\mathrm{Cu}$ & $\mathrm{Cr}$ & $\mathrm{Ni}$ & $\mathrm{Pb}$ & $\mathrm{Cd}$ & $\begin{array}{c}\mathrm{MES} \\
(\mathrm{mg} / \mathrm{l})\end{array}$ \\
\hline Febrero & 10 & 0,13 & 0,38 & 0,02 & 0,05 & 0,14 & 0,01 & 0,004 & 23 \\
\hline Marzo & 123 & 1,80 & 0,36 & 0,07 & 0,07 & 0,15 & 0,03 & 0,004 & 194 \\
\hline Abril & 83 & 1,18 & 0,43 & 0,10 & 0,07 & 0,13 & 0,06 & 0 & 155 \\
\hline Мауо & 42 & 0,65 & 0,39 & 0,05 & 0,19 & 0,12 & 0,05 & 0 & 83 \\
\hline Junio & 55 & 0,60 & 0,56 & 0,09 & 0,19 & 0,19 & 0,02 & 0,02 & 70 \\
\hline Agosto & 405,3 & 5,55 & 2,14 & 0,71 & 0,09 & 0,71 & 0,12 & 0,03 & 880 \\
\hline Septiembre & 79,6 & 0,70 & 0,39 & 0,07 & 0,09 & 0,10 & 0,04 & 0,01 & 115 \\
\hline Noviembre & 55 & 1,01 & 0,56 & 0,07 & 0,04 & 0,09 & 0,04 & 0,01 & 112 \\
\hline \multicolumn{10}{|c|}{ Remoción (\%) } \\
\hline aguas bajas & $15-53$ & $16-67$ & $19-40$ & $28-75$ & $8-64$ & $18-60$ & $10-78$ & $11-58$ & \\
\hline aguas altas & $15-67$ & $26-55$ & $15-45$ & $40-56$ & $28-65$ & $30-51$ & $35-75$ & $10-79$ & \\
\hline
\end{tabular}

MES =Material en suspensión. 
veles $(0,708 \mu \mathrm{mol} / \mathrm{l})$. El $\mathrm{Cu}$ presentó remociones entre 19 - $40 \%$ en la época de sequía y 15 - 44\% en la de lluvia. En la Figura $2 \mathrm{c}$ se observa un decaimiento brusco en las concentraciones desde 0,71 hasta $0,3 \mu \mathrm{mol} / 1$ en el mes de agosto, en los inicios de la mezcla del agua fluvial con la marina. El comportamiento presentado por el $\mathrm{Cu}$ estaría indicando que al igual que el $\mathrm{Fe}$ y $\mathrm{Mn}$, entra a la zona estudiada en forma de partículas suspendidas, aunque no se descarta que también pueden entrar en solución y luego precipitar o adsorverse en las partículas. Por otra parte es posible que las partículas coloidales de Fe y Mn estén contribuyendo a la remoción y deposición del $\mathrm{Cu}$ al inicio del mezclado de las aguas, debido a la correlación presentada entre $\mathrm{Cu}, \mathrm{Fe}$ y Mn ( $r=0,7934$ y r=0,7904), debido a que el $\mathrm{Cu}$ está asociado en los estuarios a óxidos de $\mathrm{Fe}$ y complejos húmicos (Elbaz-Poulichet et al., 1996).

Los porcentajes de remoción del $\mathrm{Cu}$ no han sido reportados con frecuencia en la literatura. Sin embargo, los reportados en este estudio son mayores que los señalados por Bewers y Yeats (1978) en el estuario de St. Lawrence. Bewers y Yeats (1983) señalan que el comportamiento del $\mathrm{Cu}$ en intervalos de salinidad entre 0,1-10 psu, es marcadamente diferente en muchas regiones estuarinas y que en algunos sitios exhibe un mínimo a salinidad de 1 psu, antes de incrementarse a un máximo a salinidad de 10 psu, y que este mínimo en las distribuciones es debido a la adsorción o cambio en la especiación del elemento en una forma que es analíticamente indetectable, tal como es el caso de los complejos órganometálicos. El comportamiento no conservativo del $\mathrm{Cu}$ ha sido reportado por Hart y Davis (1981) en el estuario del río Yarrah (Australia) y fue atribuido a la coagulación y precipitación de los complejos orgánicos del metal debido al incremento gradual del $\mathrm{pH}$ con la salinidad que ocurren en la zona de mezcla. En el río Manzanares se aprecia este mismo comportamiento en el $\mathrm{pH}$, por lo que Hurtado (1986) y León (1995) asociaron el comportamiento no conservativo del $\mathrm{Cu}$ a estos mismos mecanismos. Morse et al. (1993) en la bahía de Galveston y Zhang (1995) en los estuarios de los grandes ríos chinos, encontraron comportamiento similar para este elemento en la fracción suspendida.

Los aportes de $\operatorname{cinc}$ en la época de menor gasto del río estuvieron entre 0,56 y $2,14 \mu \mathrm{mol} / 1$ para los dos períodos, con poca variabilidad en casi todos los meses del muestreo, excepto en agosto, cuando se alcanzaron los máximos valores en las concentraciones. No
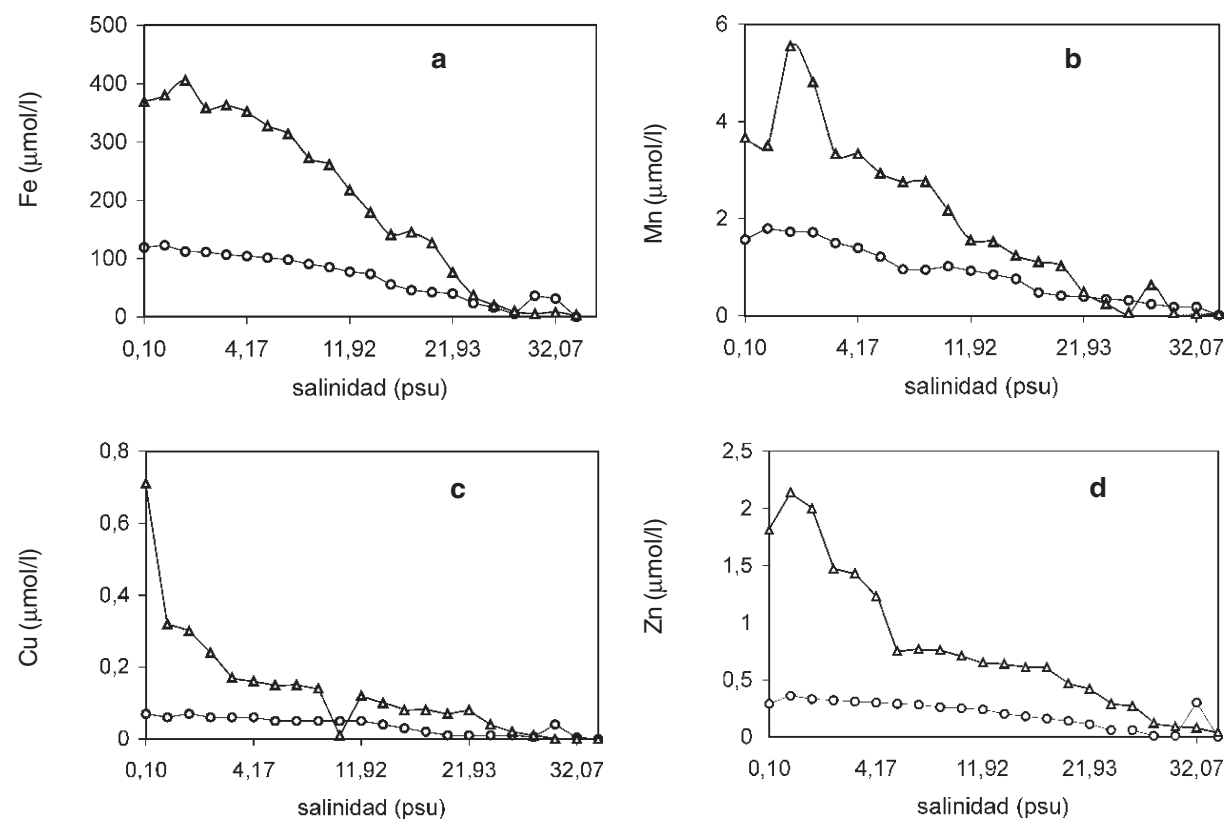

Figura 2. Diagramas de mezcla de los metales estudiados $(\mu \mathrm{mol} / \mathrm{l})$ en función de la salinidad en las la fracción suspendida de las aguas estuarinas del río Manzanares, Venezuela, durante los meses de aguas bajas (marzo — - -) y altas (agosto -- $\triangle--$ ) del año 1997.

se apreciaron grandes aportes en la zona de mezcla; sin embargo, los diagramas de mezcla Zn particulado - salinidad (Figura 2d) indican remociones a bajas salinidades, que oscilaron entre $8-64 \%$ en el período de sequía y $28-65 \%$ en el de lluvia. La concentración de $\mathrm{Zn}$ permitida para consumo humano es de $3,06 \times 10^{-4} \mu \mathrm{mol} / 1$, pero en áreas costeras y estuarios siempre son más altas debido a los aportes de las aguas industriales y urbanas (Landing et al., 1992). Este metal presentó correlación bastante significativa con el material en suspensión en este estudio ( $\mathrm{r}=0,8933)$, el $\mathrm{Fe}$ $(0,8944)$ y $\mathrm{Mn}(\mathrm{r}=0,8494)$ en agosto, lo que podría indicar que este metal esté absorbido a los óxidos coloidales de $\mathrm{Fe}$ y Mn, y que simultáneamente las partículas arcillosas podrían tener influencia en su presencia y comportamiento en las aguas del río Manzanares, ya que análisis de difracción de Rayos X realizados en este estudio reportó grandes cantidades de caolinita en el material suspendido, en donde el Zn pudo estar adsorbido, ya que es sabido que este metal esta presente en aguas naturales como resultado del desgaste de las rocas y minerales (Greenber et al., 1992).

Grandes concentraciones de $\mathrm{Zn}$ fueron reportadas por Morse et al. (1993) y Landing et al. (1995), quienes indicaron que existe una gran asociación con los granos finos del material suspendido y a los óxidos de Fe y Mn transportados por los ríos. El comportamiento no conservativo del $\mathrm{Zn}$ encontrado en esta investigación coincide con el reportado por León (1995) en este mismo río y por Zhang (1995) en el estuario Jiulongjiang. Sin embargo, un comportamiento contrario fue reportado por Apte et al. (1990) en el estuario Severn (Inglaterra), señalando aportes en la zona de mezcla a salinidades entre 0 - 15 psu, debido a los desechos industriales y domésticos.

Los niveles de níquel estuvieron por debajo de $0,2 \mu \mathrm{mol} / \mathrm{l}$ durante casi todo el período, alcanzando un máximo de $0,71 \mu \mathrm{mol} / \mathrm{l}$ en agosto, con variaciones un tanto considerables durante el transcurso de la mezcla, y remociones en un rango de 11 - 58\% en el período de sequía y $10-79 \%$ en el de lluvia. Los diagramas de mezcla $\mathrm{Ni}$ - salinidad para el mes de agosto exhiben un máximo entre 0 y 2 psu y un decrecimiento continuo con el aumento de la salinidad. El Ni, a diferencia de los otros metales evaluados, tiende a ser casi conservativo en algunos meses, presentando una alta correlación con la salinidad (febrero, r=0,9059; abril, $\mathrm{r}=0,9532$; septiembre, $\mathrm{r}=0,8230$; noviembre, $r=0,9363)$. Tal característica puede ser debida a la poca labilidad que presenta este metal en los sistemas estuarinos. Los niveles de $\mathrm{Ni}$ admisibles por la legislación venezolana son de $3,41 \times 10^{-2} \mu \mathrm{mol} / 1$ para aguas a ser descargadas a los medios marinos - costeros, mientras que no existen limites establecidos para las aguas destinadas al consumo humano. Los valores normales en agua de mar son de $1,70 \times 10^{-3}$ a $4,43 \times 10^{-2} \mu \mathrm{mol} / 1$. 

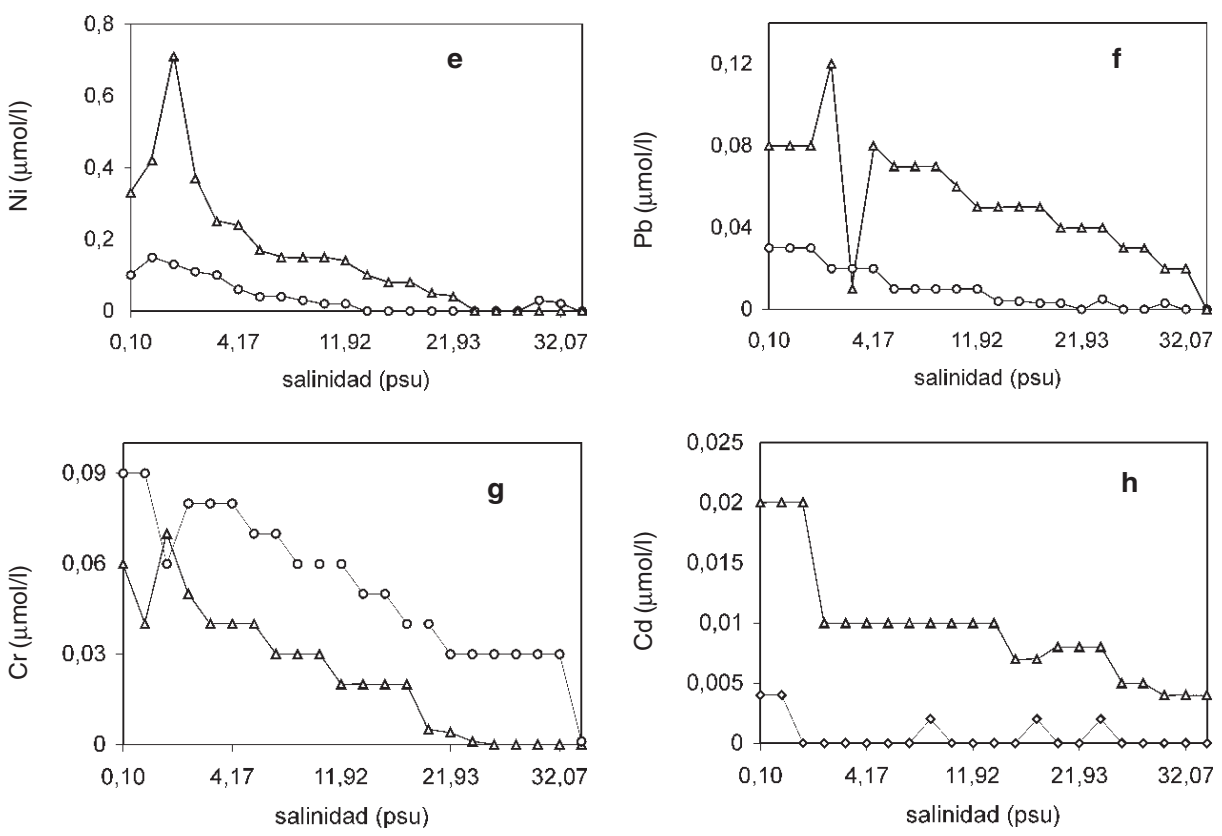

Continuación Figura 2. Diagramas de mezcla de los metales estudiados ( $\mu \mathrm{mol} / \mathrm{l}$ ) en función de la salinidad en la fracción suspendida de las aguas estuarinas del río Manzanares, Venezuela, durante los meses de aguas bajas (marzo - - -) y altas (agosto -- $\triangle--$ ) del año 1997.

Las remociones que presenta el $\mathrm{Ni}$ en los primeros inicios de mezcla en las aguas superficiales del Manzanares posiblemente están influidas por la floculación desde los óxidos de $\mathrm{Fe}$ y $\mathrm{Mn}$, puesto que ese metal puede adherirse a la superficie de los óxidos coloidales de estos dos elementos, así como a silicatos y carbonatos (Moore y Ramammorthy, 1984). Estas observaciones se aprecian en el río Manzanares por la correlación positiva que presenta con el Fe y $\mathrm{Mn}$ en el mes de agosto $(\mathrm{r}=0,7104 \mathrm{y}$ 0,8628), confirmando los estudios de Zhang (1995) quien señaló que el Ni manifiesta remoción desde el particulado y desorción desde la fase sólida suspendida debido a los procesos inorgánicos. Estas observaciones se aprecian en este estudio en la zona estuarina del río Manzanares (Figura 2e) y se confirman con la correlación del $\mathrm{Ni}$ con el material en suspensión ( $\mathrm{r}=0,8836)$. Morse et al. (1993), de igual manera, reportaron buena correlación del $\mathrm{Ni}$ con los óxidos de Fe y la fracción fina de los granos suspendidos en la bahía de Galveston.

La alta correlación presentada entre el Ni y la salinidad en algunos meses, refleja la poca labilidad que presenta este metal en los sistemas estuarinos, lo que corrobora los señalamientos de Zhang (1995) en sus estudios. El comportamiento no conservativo experimentado por el $\mathrm{Ni}$ en este estudio ha sido reportado en el estuario Delaware (USA), debido a floculación geoquímica y a procesos de desorción desde el sedimento a salinidades entre 0-15 psu (Sharp et al., 1982; Apte et al., 1990). Sin embargo, conservatividad de este elemento fue reportada en el estuario del río Lena (Martin et al., 1993). El comportamiento anormal del $\mathrm{Ni}$ es bastante complejo en muchos estuarios, debido a que él participa en los procesos biogeoquímicos.

Los aportes de plomo en el río estuvieron entre 0,1 y $0,12 \mu \mathrm{mol} / 1$. La relación entre el $\mathrm{Pb}$ y salinidad (Figura 2f) refleja comportamiento no conservativo con remociones a bajas salinidades en casi todos los muestreos y gran conservatividad en el mes de agosto $(r=0,8716)$. Estas remociones oscilaron en un rango de $10-78 \%$ en el período de sequía y 33 - $75 \%$ en el de lluvia. La legislación venezolana establece concentraciones limites de $\mathrm{Pb}$ de $2,42 \mu \mathrm{mol} / 1$ para las aguas de descargas a los medios marino - costero, y de $0,25 \mu \mathrm{mol} / 1$ para las aguas destinadas al consumo humano (Senior, 1994).

Las concentraciones se incrementaron con el período de lluvia (Figura 2f). Sin embargo, los aportes durante el período de mayor gasto del río (agosto), son casi iguales a los del período de menor flujo del río, lo que estaría sugiriendo que el metal esta siendo introducido por una fuente diferente al drenaje del río y que posiblemente la deposición de partículas de $\mathrm{Pb}$ sobre el material suspendido, generada por las actividades antropogénicas, tales como la combustión de la gasolina utilizada por las embarcaciones que transitan por esa zona y por la presencia de la bomba de gasolina en su desembocadura, que genera especies como di, tri, y tetra alquil y tetra metil de $\mathrm{Pb}$ sean los responsables, como lo señalan en sus investigaciones Landing et al.(1992) y Sadid (1992), quienes indican que el $\mathrm{Pb}$ está asociado en adsorción con los óxidos de Fe y a la fracción fina del material suspendido.

La remoción del elemento del material suspendido podría deberse a procesos de floculación y/o precipitación desde los óxidos coloidales de Fe y $\mathrm{Mn}$, debido a que en la superficie agua de río-mar de los medios estuarinos, el $\mathrm{Pb}$ tiende a precipitar por su gran reactividad y los cambios de $\mathrm{pH}$ y fuerza iónica (Sadid, 1992; Morse et al., 1993). La correlación presentada por el $\mathrm{Pb}$ con el material en suspensión ( $\mathrm{r}=0,8817)$, Fe $(0,8467)$ y $\mathrm{Mn}(0,8475)$, indica gran asociación a partículas coloidales suspendidas, lo cual se corrobora con los estudios realizados en el estuario del río Yarrah, Australia (Hart y Davis, 1981) y en el río Rhine, Alemania (De Groot et al., 1976). El comportamiento de este metal no esta muy bien documentado en la literatura (Elbaz-Poulichet et al., 1996); sin embargo algunas remociones y comportamiento no conservativo han sido encontrados en el estuario Gironde (ElbazPoulichet et al.,1984) y Bang Pakog (Windom et al.,1988). Las concentraciones de $\mathrm{Pb}$ encontradas en este estudio deben tenerse en consideración, puesto que, este metal no cumple ninguna función biológica, y puede en muy pequeñas concentraciones adsorberse en la superficie de algunas especies marinas (Song y Müller, 1995), pudiendo causar al mismo tiempo problemas adversos a la biota acuática (Wong et al., 1978) y a la población de Cumaná, dada la gran capacidad para crear problemas de retardo mental y desordenes metabólicos en los seres humanos, especialmente en los niños (Nriagu, 1988; Silvany-Neto et al., 1989).

El cromo osciló entre 0,19 y $0,09 \mu \mathrm{mol} / 1$ en los meses de mayor caudal del río. Como se puede apreciar en la Figura 2g, el Cr tuvo un comportamiento diferente al de los otros metales estudiados, ya que a diferencia de aquellos, sus concentraciones disminuyeron con el aumento del caudal del río. Las remociones oscilaron entre $28-75 \%$ en el período de sequía y 40 - 56\% en el de lluvia. Los niveles de $\mathrm{Cr}$ (VI) establecidos por las normas internacionales son de 0,058 a 0,077 $\mu \mathrm{mol} / 1$ (Greenber et al., 1992). El $\mathrm{Cr}$, a diferencia de los otros metales, presentó su máximo en el período de aguas bajas. El incremento en los primeros me- 


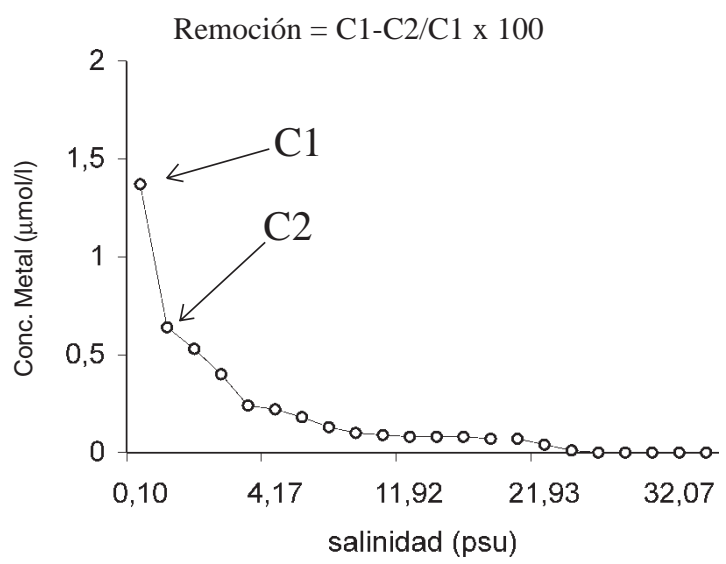

Figura 3. Diagrama explicativo para cálculo de remociones de metales en sistemas estuarinos. (concentración -O-)

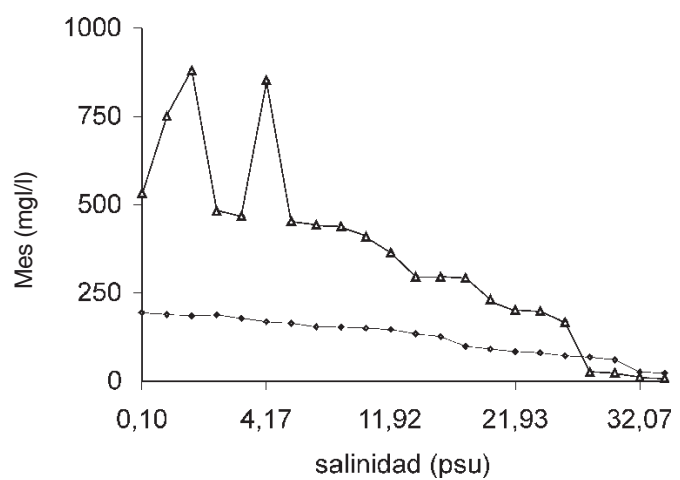

Figura 4. Diagramas de mezcla del material en suspensión (MES) en función de la salinidad en la fracción suspendida de las aguas estuarinas del río Manzanares, Venezuela, durante los meses de aguas bajas (marzo $\longrightarrow-$ ) y altas (agosto $--\triangle--$ ) del año 1997. ses del año puede ser debido a la acumulación de partículas que son vertidas por las actividades antropogénicas, que se acumulan por el mayor tiempo de residencia de las aguas.

Los mecanismos de floculación y precipitación son los principales factores que propician la deposición del Cr, como señaló León (1995) en investigación realizada en la misma zona de este estudio. Los decrecimientos posteriores del $\mathrm{Cr}$ con el aumento del caudal del río posiblemente son generados por procesos de dilución parecidos a los reportados en río Rhöne, Francia (ElbazPoulichet et al., 1996) quienes señalaron que las concentraciones de algunos metales como el Cr tienden a estar inversamente relacionadas con la descarga del río, atribuyéndose ésto a los mecanismos de dilución y a los impactos antropogénicos. La alta correlación encontrada entre el $\mathrm{Cr}$ y el material en suspensión $(\mathrm{r}=0,8835)$, concentración de Fe $(r=0,9000)$ y Mn $(r=0,9404)$ sugeriría que este elemento es introducido al sistema estuarino del Manzanares en forma de partículas y posiblemente adsorbido a la superficie de los óxidos de $\mathrm{Fe}$ y $\mathrm{Mn}$, ya que en muchos estudios, como por ejemplo, el de Morse et al. (1993) se señala, que el $\mathrm{Cr}$ exhibe muy buena correlación con la fracción fina y que la desorción ocurre por adsorción y desegregación de coloides. La asociación a partículas reseñadas por estos autores y por otros como Gibbs (1977) en el río Amazonas, parecen confirmarse en este estudio. Estudios más recientes confirman que este metal esta asociado al carbono orgánico y a los óxidos de $\mathrm{Fe}$, y que es liberado desde ellos debido a las condiciones de óxido-reducción (Landing et al.,1995). Los resultados de este estudio confirman el comportamiento no conservativo reportado en el río Manzanares (Hurtado, 1986; León, 1995).

Los niveles de cadmio encontrados fueron $0,02 \mu \mathrm{mol} / 1$ en el período de sequía y $0,028 \mu \mathrm{mol} / \mathrm{l}$ en la etapa de mayor caudal del río. Desde el inicio de los muestreos los valores tuvieron una gran fluctuación; en el período de menor gasto del río las concentraciones de este elemento fueron muy bajas, e incluso llegó a no detectarse en algunos meses; sin embargo posteriormente, con el inicio del período de lluvia, las concentraciones se incrementaron hasta alcanzar su máximo en agosto. En la Figura $2 \mathrm{~h}$ se observa remociones a bajas salinidades en un rango de $18-60 \%$ en la época de sequía y $30-51 \%$ en la de lluvia, y poca variabilidad en el comportamiento entre 16 y 30 psu.

Las concentraciones de $\mathrm{Cd}$ en aguas naturales y de consumo humano varían por debajo de $8,74 \times 10^{-4}$ $\mu \mathrm{mol} / \mathrm{l}$. La legislación venezolana establece valores limites de $1,75 \mu \mathrm{mol} / \mathrm{l}$ para las aguas de descargas al medio marino costero y de $0,044 \mu \mathrm{mol} / 1$ para las destinadas al consumo humano. En el presente estudio las concentraciones encontradas $(0,028 \mu \mathrm{mol} / \mathrm{l})$, si bien están por debajo de las reglamentadas su presencia es ubicua y no deja de ser preocupante, ya que es reflejo de impactos antropogénicos. González y Ramírez (1995) señalan al Cd junto al $\mathrm{Pb}, \mathrm{Cu}$ y $\mathrm{Zn}$ como buenos indicadores de contaminación generada por el hombre, debido a que sus concentraciones en el medio ambiente son reflejo de las actividades humanas y fuentes industriales. Aún en el orden de concentración encontrado en este estudio, el $\mathrm{Cd}$ puede ser altamente tóxico, pudiendo ser res- ponsable de los cambios adversos en la biota y en las arterias y riñones de los humanos (David y Phillips, 1995). La disminución en las concentraciones del $\mathrm{Cd}$ con la salinidad (Figura 2h), al igual que los otros metales evaluados, posiblemente se debe a procesos de floculación desde los óxidos de Fe y Mn, debido a los cambios de $\mathrm{pH}$ que ocurren en esta región del ecosistema estudiado, ya que este elemento se encontró bien correlacionado con material suspendido $(\mathrm{r}=0,9016)$ $\mathrm{y}$ con el $\mathrm{Fe}(\mathrm{r}=0,7518)$. El $\mathrm{Cd}$ ha sido asociado a los óxidos de $\mathrm{Fe}$ y a la fracción fina del suspendido (Sadid, 1992; Morse et al., 1993; Zhang, 1995), aunque también es asociado frecuentemente con los compuestos de Zn (Sadid, 1992). En este estudio se encontró una buena correlación del $\mathrm{Cd}$ con el $\mathrm{Zn}(\mathrm{r}=0,8456)$, lo que corrobora las observaciones hechas por esos autores.

Los resultados coinciden con los señalados por Song y Müller (1995), quienes indicaron que gran cantidad de $\mathrm{Cd}$ se encuentra adherida a los óxidos de $\mathrm{Fe}$ y $\mathrm{Mn}$ en el río Neckar. El comportamiento no conservativo observado para este elemento coincide con las observaciones reportadas en el estuario del río Yarrah, Australia (Hart y Davis,1981), en el estuario Delaware, USA (Sharp et al., 1982) y en el río Mississippi (Shiller y Boyle, 1991), en las cuales fue asociado a procesos de floculación geoquímica.

Los análisis de rayos $\mathrm{X}$ realizados a las muestras obtenidas mostraron que el material en suspensión arrastrado por el río Manzanares contiene arcillas (caolinita), carbonatos y silicatos. Los mayores valores se encontraron en el extremo fluvial en los meses de mayor 
pluviosidad, especialmente en agosto, cuando se alcanzaron valores de $880 \mathrm{mg} / \mathrm{l}$ (Tabla II). Los valores oscilaron entre 112 y $880 \mathrm{mg} / \mathrm{l}$ en el extremo fluvial en la época de lluvia, y entre 23 y 194 mg/l en el período de sequía.

El alto valor de $194 \mathrm{mg} /$ 1 que se obtuvo en el mes de marzo (aguas bajas) está asociado al material sólido orgánico de origen antropogénico que es vertido en el sector, el cual se concentra en el período de menos gasto del río y mayor tiempo de residencia de las aguas, o por la resuspensión de partículas desde los sedimentos de fondo. Sin embargo los incrementos en el período de aguas altas esta asociado la erosión de los suelos en la cuenca alta del río Manzanares (Aguilera et al., 1985)

Este material presentó un comportamiento no conservativo con remoción a salinidades entre 0 y 5 psu y disminuciones en las concentraciones con el incremento de la salinidad (Figura 4). Las remociones a bajas salinidades y disminuciones de las concentraciones del material en suspensión con el aumento de la salinidad sugieren que posiblemente los cambios de la fuerza iónica que tienen lugar en la interfase agua de río mar y el pH son los responsables de los procesos de floculación que rigen este comportamiento en este material. Según Burton (1976) las cargas netas de las partículas en agua dulce son negativas y tienden a conservarse por los efectos de repulsión entre cargas de igual signo que se superponen a las fuerzas de Van der Waals, pero que al ingresar a las aguas salinas, las fuerzas repulsivas se reducen, produciendo desestabilización de los coloides, aumentando la tendencia a flocular, situación que se favorece por el movimiento de las partículas y el incremento del material en suspensión.

Comportamientos similares de remoción a bajas salinidades fueron reportados en el estuario de Yakina, USA (Callaway et al., 1988), en el río Orinoco (Salazar, 1989), el Manzanares (Senior y Godoy, 1991) y en el río Rhöne, Francia (Elbaz-Poulichet et al., 1996), atribuyéndose estas manifestaciones a la floculación y precipitación del material suspendido.

\section{Conclusiones}

1. Las concentraciones de todos los metales estudiados están muy por encima de los valores reglamentados por la legislación venezolana para aguas de consumo e incluso para aguas de descarga a los cuerpos de aguas litorales. Esto podría estar afectando la estabilidad del ecosistema poniendo en peligro la vida de la biota acuática y de la población asentada en los márgenes del río Manzanares.

2. Los metales estudiados y el material en suspensión presentaron un aumento considerable en sus concentraciones en el período de aguas altas debido al arrastre de la gran cantidad de material en suspensión arrastrada por el río. Sin embargo, los aportes del río para $\mathrm{Cd}, \mathrm{Cr}$ y $\mathrm{Pb}$ no son continuos, lo que sugiere que estos metales podrían estar siendo introducidos desde fuentes alternas como pudieran ser, el astillero y la bomba de gasolina ubicados en los alrededores de la desembocadura, en donde dichos elementos forman parte de las aleaciones $(\mathrm{Pb}, \mathrm{Cd}$ y $\mathrm{Cr})$ y de los compuestos antidetonantes de la gasolina $(\mathrm{Pb})$.

3. La alta correlación lineal que presentaron los metales con el material en suspensión, sugiere que una de las causas por las cuales estos elementos entran al sistema estuarino es la asociación a las partículas suspendidas, aunque la resuspensión desde los sedimentos de fondo, la entrada en solución del metal y posterior precipitación y /o adsorción en las partículas no está descartada.

4. Una de las causas de las remociones a bajas salinidades apreciadas en los metales estudiados y el material en suspensión es la presencia de procesos de floculación, adsorción a partículas sólidas y precipitación del material en suspensión a la cual esta asociado el metal. De igual manera la deposición a altas salinidades de los metales está afiliado a la precipitación en forma de oxihidróxidos cuando el $\mathrm{pH}$ se incrementa gradualmente desde el agua fluvial hasta la mezcla de esta con el agua marina, debido a las sales básicas disueltas.

\section{AGRADECIMIENTOS}

Los autores agradecen al personal Técnico del Departamento de Oceanografía del Instituto Oceanográfico de Venezuela que colaboró en este estudio. Al MsC. Julio Rodríguez del Instituto Limnológico de la UDO, por las correcciones y sugerencias realizadas. Esta investigación contó con el apoyo financiero del Consejo de Investigación de la UDO, a través de los proyectos C.I.51801-0693/94; CI-5-019-006921/94.

\section{REFERENCIAS}

Aguilera De León L, Rojas L (1976) La Ictiofauna del complejo hidrográfico del río Manzanares, Edo. Sucre. Venezuela. Lageda., 37-38: 23-35.
Aguilera D, Lastra R, Betancourt M (1985) Rescate del río Manzanares. Informe preliminar de la comisión nombrada por el Consejo Municipal del Distrito Sucre (Estado Sucre) sobre la conservación, mejoramiento y defensa del Manzanares, su cuenca y el área bajo su influencia. $46 \mathrm{pp}$

Alvarado E (1976) Algunas observaciones sobre la concentración de fosfatos en el río Manzanares. Tesis de Pregrado. Lic. Educación mención Química. Escuela de Humanidades y Educación. Universidad de Oriente, $\mathrm{Cu}$ maná, Venezuela. 34pp.

Apte S, Gadner M, Gunn A, Ravenscroft J, Vale J (1990) Trace metals in the Severn estuary: A reappraisal. Mar.Poll.Bull., 21 (8): 393-396.

Bewers J, Yeats P (1978) Trace metals in water of a partially mixed estuary. Estuar. Coast. Mar.Sci., 1: 147-162.

Bewers J, Yeats P (1983) Behaviour of trace metal during estuarine mixing. En: River imputs to ocean system. United Nations, New York. pp. 103-115.

Burton J (1976) Basic properties and processes in estuarine Chemistry. Burton J, Liss P (eds), 1a. Edición. Academic Press, USA: $1-36$.

Callaway R, Spech D, Ditsworth G (1988) Manganese and suspend matter in the Yaquina estuary. Oregon. Estuar. 11 (4): 217-225.

Carpenter J, Bradford W, Grand V (1975) Processes affecting the composition of estuarine waters $\left(\mathrm{H}_{2} \mathrm{CO}_{3}, \mathrm{Fe}, \mathrm{Mn}, \mathrm{Cu}, \mathrm{Ni}, \mathrm{Cr}\right.$ and Co). En: Estuar. res. Cron. L. (De). Academic Press, New York. Vol. 1: 188-214.

David J, Phillips H (1995) The chemistries and environmental fates of trace metals and organochlorines in aquatic ecosystems. Mar. Poll. 31 (12): 4-12.

DeGroot A, Salomons W, Allersma E (1976) Processes affecting Heavy metals in estuarine sediments. En: Burton $\mathrm{J}$, Liss $\mathrm{P}$ (eds.) Estuarine Chemistry. Academic Press Inc. USA. pp. 131-157.

Duinker S, Nolting R (1978) Mixing, removal and mobilization of trace metals in the Rhine estuary. Neth. J. of Sea Res. 12: 205223.

Elbaz-Poulichet F, Garnier J, Guan M, Martin J, Thomas A (1996) The conservative behavior of trace metals $(\mathrm{Cd}, \mathrm{Cu}, \mathrm{Ni}$ and $\mathrm{Pb})$ and as in the surface plume of stratified estuaries: Example of the Rhöne River (France). Estuar. Coast. and Shelf. Sci. 42: 280-310.

Fernández E (1984) Contaminación de los ríos Guasdua y Manzanares. Edo. Sucre, Venezuela. Bol. Inst. Oceanograf. Univ. Oriente. $23(1-2): 113-128$.

Gibbs R (1977) Transport phases of transition metals in the Amazon and Yukon Rivers. Geol. Soc. of Amer. Bull. 88 (5 - 8): 829843.

Godoy G (1991) Estudio espacio-temporal de los parámetros fisicoquímicos y biológicos en la zona estuarina del río Manzanares (Cumaná- Venezuela). Trabajo de grado de M.Sc. en Ciencias Marinas. Inst. Oceanográf. de Venezuela, Universidad de Oriente, Cumaná, Venezuela. 185pp.

González H, Ramírez M (1995) The effect of nickel mining and metallurgical activities on the distribution of heavy metals in Levisa Bay, Cuba. Jour. Geochem. 52: 183 $-192$

Greenber A, Clesceri L, Eaton A (1992) Standard Methods for the Examination of Water and Wastewater. 18 Edition. APHA-AHWA. USA. $3060 \mathrm{pp}$. 
Hart B, Davis S (1981) Trace metals speciation in the freshwater and estuarine regions of Yarrah River (Victoria). Estuar. Coast. Shelf. Sci. 12: (4): 353-374.

Hurtado C (1986) Comportamiento de algunos metales en sistemas estuarinos. Trabajo de grado de M.Sc. en Ciencias Marinas. Inst. Oceanográf. de Venezuela, Universidad de Oriente, Cumaná, Venezuela.126 pp.

Iabichella M (1993) Evaluación bacteriológica del sector marino-costero San Luis-Guapo, Cumaná. Venezuela; según los criterios para las aguas de contacto humano total y parcial. Trabajo de grado de M.Sc. en Ciencias Marinas. Inst. Oceanográf. de Venezuela, Universidad de Oriente, Cumaná, Venezuela. 300 pp.

Landing W, Cutter G, Dalzier J, Flegal A, Powell R, Schidt D, Shiller A, Westerlund S, Resing J (1995) Analytical intercomparison results from the 1990' Intergovernmental Oceanographic Commission open-ocean baseline survey for trace metals: Atlantic ocean. Mar.Chem. 49: 253-265.

Landing W, Lyons W, Orem W (1992) Nutrient cycling and the biochemistry of $\mathrm{Mn}, \mathrm{Fe}$, and $\mathrm{Zn}$ in jelly fish lake, Palao. Limnol. Oceanograf. 36: 515-525.

León I (1995) Comportamiento y distribución de los metales pesados (Fe, Cu, Cd, Mn, $\mathrm{Cr}, \mathrm{Ni}, \mathrm{Zn}$ y $\mathrm{Pb}$ ) en la cuenca baja y pluma del río Manzanares (Cumaná - Venezuela). Trabajo de grado de MSc. en Ciencias Marinas. Inst. Oceanográf. de Venezuela, Universidad de Oriente, Cumaná, Venezuela. 214pp.

Liss P (1976) Conservative and non-conservative behavior of dissolved constituent during estuarine mixing. En: Burton J, Liss $\mathrm{P}$ (eds). Estuarine Chemistry. Academic Press. Inc. USA. 229 pp.

Márquez A (1997) Comportamiento y distribución de algunos metales pesados en fracciones disueltas y particuladas en aguas superficia- les del río Manzanares. Edo. Sucre, Venezuela). Trabajo de Grado en Lic. Química. Dep. Química de la Universidad de Oriente, Cumaná, Venezuela. 141pp.

Martin J, Guan D, Elbaz-Poulichet F, Thomasy A, Gordev V (1993) Preliminary assessment of the distribution of some trace element (As, $\mathrm{Cd}, \mathrm{Cu}, \mathrm{Fe}, \mathrm{Ni}, \mathrm{Pb}$ and $\mathrm{Zn}$ ) in a pristine aquatic environment in the Lena Rive estuary (Russia). Mar. Chem, 43: 185-199.

Moore J, Ramammorthy S (1984) Heavy Metal in Natural Waters. Pergamon Press. Gran Bretaña. 268 pp.

Morris A, Bale A (1979) Effect of rapid precipitation of dissolved $\mathrm{Mn}$ in river waters on estuarine Mn distributions. Nat. 229: 303 327.

Morse J, Presley B, Taylor R, Benoit G, Santschi P (1993) Trace metal chemistry of Galveston Bay: water, sediments and biota. Mar. Envir. Res. 36: 1-37.

Murray J, Gill G (1978) The geochemistry of iron in Puget Sound. Geochim. Cosmochim. Acta.42: 9-20.

Nriagu J (1988). A silent epidemic of environmental metal poisoning. Envir. Poll. 50: 139-61.

Rainbow P (1995) Biomonitoring of heavy metal availability in the marine environment. Mar. Pull. Bull. 31 (4-12): 183-192.

Sadid M.(1992) Toxic metal chemistry in marine environments. Pergamon Press. Gran Bretaña. $389 \mathrm{pp}$.

Salazar J (1989) Condiciones hidrogeoquímicas de la región estuarina - deltáica del Orinoco durante el mes de noviembre de 1985 Trabajo de grado de MsC en Ciencias Marinas. Inst. Oceanográf. de Venezuela, Universidad de Oriente, Cumaná, Venezuela. $128 \mathrm{pp}$

Salinas J, Ruiz J, Frances G (1996) Heavy metal levels in intertidal sediments and biota from the Bidasoa estuary. Mar. Pull. 32: 1 69-71pp.
Senior W. (1994). Diagnóstico ambiental del río Manzanares. Informe técnico. Departamento. de Oceanografía, Universidad de Oriente, Cumaná, Venezuela UDO. 22pp.

Senior W (1995) Manual de Métodos de Análisis de Agua de Mar. Instituto Oceanograf. de Venezuela, Universidad de Oriente, $\mathrm{Cu}$ maná, Venezuela. 22pp.

Senior W, Godoy G (1991) Estudio fisicoquímico del río Manzanares (Cumaná-Venezuela). Bol. Inst. Oceanográfico. UDO. 29 (1-2): 160-172.

Sharp J, Culberson Ch, Church T (1982) The Chemistry of the Delaware estuary. General considerations. Limnol. Oceanogr. 27 (6): 1015-1028.

Shiller A, Boyle E (1991) Trace metals in Mississippi River. Delta out region: behavior at low discharge. Geochimic. Cosmochim. Acta. 35. 32: (41). 3251.

Silvany-Neto A, Carvalho M, Chavez M, Brando A,Tavares T (1989) Repeated surveillance of lead poisoning among children. Sci. Environ. 78 : 179-186.

Song Y, Müller G (1995) Biogeochimical cycling of nutrients and trace metals in anoxic freshwater sediments of the Neckar River. Germany. Mar. Freshwater. Res. 46: 237-43.

Wells M, Mayer L (1991) Variations in the chemical lability of iron in estuarine coastal and shelf waters and its implication for phytoplankton. Mar. Chem. 32: 195-210.

Windom H, Smith R, Dharmavajni S, Wattayakorn G (1988) Trace metals transport in a tropical estuary. Mar. Chem. 24: 243-304.

Wong P, Silverberg B, Chau G, Hodson P (1978) Lead and aquatic biota. En: Nriagu, $\mathrm{J}$ (Ed.) The Biochemistry of Lead in the Environment. Elsevier, Amsterdam. 279pp.

Zhang J (1995) Geochemistry of trace metals from Chinese rivers/estuary systems. Estuar. Coast. and Sci. 41: 631-658. 